\title{
Lactic acid recovery profiles following exhaustive arm exercise on a canoeing ergometer
}

\author{
S. J. Baker PhD and N. King BSc \\ Sport Health and Physical Education, University College of North Wales, Bangor, UK
}

\begin{abstract}
In this study lactate removal rates were monitored in ten subjects during a $30-\mathrm{min}$ recovery period following exhaustive arm exercise. Each subject experienced three recovery regimens on different occasions in random order. One recovery period consisted of supine rest while the other two were active, one incorporating low intensity arm exercise and the other consisting of low intensity leg exercise. Lactate clearance rates showed a significant difference between the recovery regimens, and a posteriori test indicated a significant difference between leg exercise and arm exercise in the recovery period and also between leg exercise and rest. There was no significant difference in lactate clearance between arm exercise and rest in the recovery period.
\end{abstract}

Keywords: Slalom canoeing, lactate removal, arm exercise

In the sport of slalom canoeing a competitor must manoeuvre his kayak through a series of 'gates' suspended over rapid and agitated water. The objective is to reach the finish in the shortest possible time and to avoid any time penalties which are incurred if he contacts a 'gate'. Recent developments in the sport have shortened the courses and reduced individual run times to under $2 \mathrm{~min}$ and as a result it might be suggested that the relative contribution of aerobic and anaerobic glycolysis has also been modified from that required for previously longer events. In a world class event when courses were longer than is suggested here, it has been shown that anaerobic involvement as manifested by postcompetition serum lactate values $\left(16.2 \mathrm{mmoll}^{-1}\right)$ was considerable ${ }^{1}$.

The removal of lactic acid after exercise may be important during athletic competitions since lactic acid has been shown to inhibit the rate of glycolysis ${ }^{2}$. However, in slalom canoeing, where a competitor is entitled to two runs down the course, the interval between runs is often several hours and it might be expected that lactic acid levels would reach resting levels between runs ${ }^{3}$. Perhaps the rate of lactate clearance is more important for the canoeist during

Address for correspondence: Dr S. Baker, SHAPE, Ffriddoedd Building, University College of North Wales, Bangor, Gwynedd, UK

(C) 1991 Butterworth-Heinemann Ltd.

0306-3674/91/030165-03 training bouts where regimens are often intense and long lasting.

Many investigators have shown that the rate of lactic acid removal occurs more rapidly during aerobic recovery exercise than at rest ${ }^{4-6}$. Moreover, after a running bout it has been demonstrated that the intensity of recovery exercise is related to the rate of lactic acid removal. Continuous recovery exercise was shown to be more effective in removing lactate than intermittent recovery exercise ${ }^{3}$. A similar relationship between lactate removal and intensity of recovery exercise was demonstrated more recently in the sport of rowing where it was shown that during the recovery period when athletes continued rowing at $40 \%$ of maximum speed there was a greater rate of lactate removal than when recovering using a $60 \%$ speed $^{7}$. In these studies the mode of exercise during recovery incorporated the same muscle groups as those used during lactate accumulation. Although some lactate which has accumulated from intense exercise may be removed by various organs such as the brain, myocardium, renal medulla and liver, the majority is removed by skeletal muscle ${ }^{7}$.

In the sport of slalom canoeing, upper body musculature is involved in intense isotonic activity while the lower body is involved in isometric synergic activity. Following an event it is not always convenient for the competitor to remain in his kayak and promote lactate clearance through continued but less intense paddling activity. It would be very useful for canoeing coaches to be able to influence the rate of lactate clearance in their athletes using activity other than canoeing.

The purpose of this study was to compare the rates of lactate clearance during different modes of recovery exercise.

\section{Methods}

\section{Subjects}

Ten healthy male university students who had no experience of competitive canoeing participated in the study. Their mean(s.d.) characteristics were: age 22.0(3.9) years; weight 70.3(5.6) kg; and height $170.6(5.6) \mathrm{cm}$.

All subjects became accustomed to working on an electronically controlled (LODE) canoeing ergometer $^{8}$. Before exercise the dominant hand of each 
subject was gently warmed by immersion in warm water for 3-4 min. The hand was dried and the subject worked on the ergometer against an initial resistance of $50 \mathrm{~W}$ at a rate of $60-70$ revs per min, with an increment of $40 \mathrm{~W}$ every $2 \mathrm{~min}$ until volitional exhaustion.

Each subject was assigned randomly to one of three recovery protocols and each subject experienced all recovery protocols following exercise on subsequent occasions. A minimum period between exercise bouts for each subject was $48 \mathrm{~h}$.

\section{Recovery protocols}

Each recovery period lasted for $30 \mathrm{~min}$ during which blood samples were taken from a finger tip of the dominant hand and assayed for lactate content.

Three modes of recovery were used:

1. Passive recovery where the subject rested in a supine position with a minimum of movement.

2. Active leg recovery where subjects pedalled a cycle ergometer for $30 \mathrm{~min}$ against a resistance of $1.5 \mathrm{~kg}$ at a steady rate of 30 revs per min.

3. Active arm recovery where the subject worked on the arm ergometer against a resistance of $40 \mathrm{~W}$ at 40 revs per min. Work intervals of $2.5 \mathrm{~min}$ were interspersed with rest periods of $2.5 \mathrm{~min}$ for the duration of the recovery period.

\section{Lactate sampling}

An Analox GM7 analyser using lysing capillary tubes containing heparin, fluoride and nitrite (Analox Instruments, Hammersmith, London) was used to determine lactate concentrations.

After exercise to volitional exhaustion, blood samples were taken at $0.5,2$ and $5 \mathrm{~min}$ and then at each 5-min interval for the recovery period. Mean results of subject lactate concentrations at each sampling point over three modes of recovery exercise were treated statistically using a repeated measures two-way analysis of variance (ANOVA).

\section{Results}

Mean(s.d.) blood lactate values were calculated and since these were quite variable throughout all three recovery regimens, concentrations in each recovery regimen were also expressed relative to the maximum mean lactate value recorded (Table 1). These data are illustrated in Figure 1.

It is interesting to note from Table 1 that the time at which peak mean lactate values were recorded was the same for the arm exercises and resting recovery regimens, i.e. $5 \mathrm{~min}$ after exhaustive exercise, but during the leg exercise recovery period peak mean lactate was recorded at $2 \mathrm{~min}$. From the same table it can be seen that there was also a similarity in the peak values of lactate recorded between the resting condition and the arm recovery regimen, $11.9 \mathrm{mmoll}^{-1}$ and $11.6 \mathrm{mmoll}^{-1}$ respectively. The peak mean value recorded during leg exercise recovery was lower at $9.9 \mathrm{mmoll}^{-1}$.

After $30 \mathrm{~min}$ of recovery, mean lactate values for both the resting and arm exercises $\left(5.6 \mathrm{mmoll}^{-1}\right.$, and $4.3 \mathrm{mmoll}^{-1}$ respectively, Table $1 ; 47 \%$ and $37.1 \%$

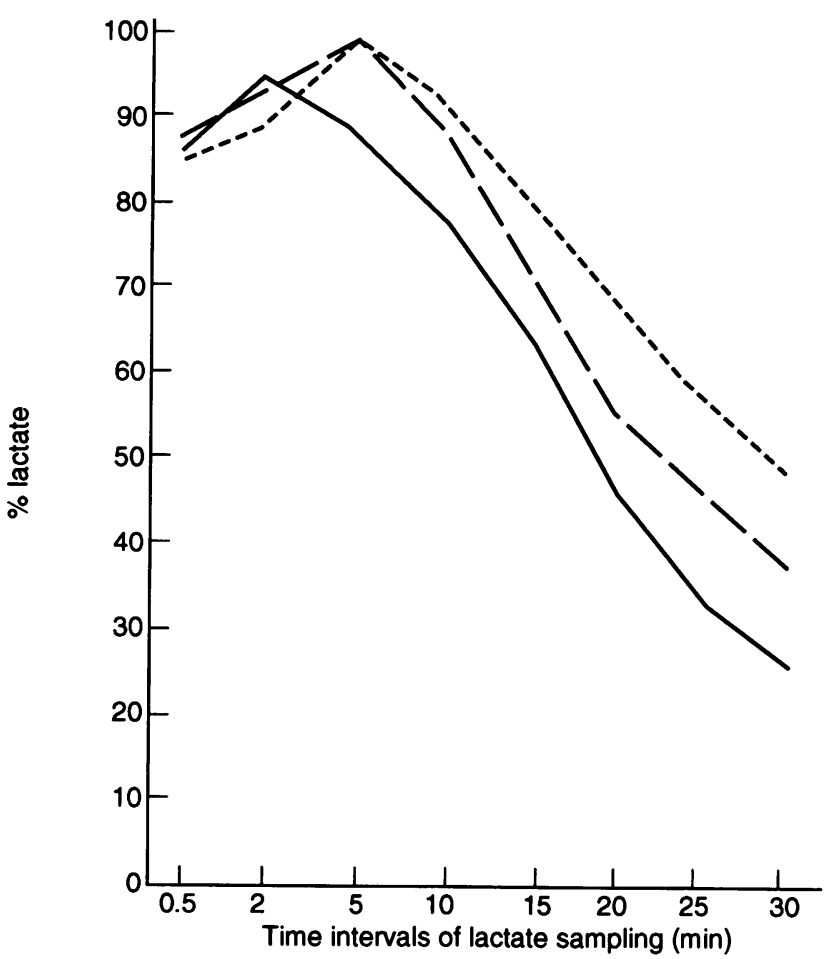

Figure 1. Lactate profiles of three recovery regimens: rest, arms and legs recorded over $30 \mathrm{~min}$. Results are expressed as a percentage of mean maximum lactate values $\left(\mathrm{mmol}^{-1}\right)$ for each condition: -...- , rest; - arms; - legs

respectively, Figure 1) were above $4 \mathrm{mmoll}^{-1}$, which is widely used as the point at which lactate production exceeds lactate clearance rates, whereas after $30 \mathrm{~min}$ of gentle leg exercise approximately $75 \%$ of lactate had been removed and lactate values were approaching normal resting levels, $2.5 \mathrm{mmoll}^{-1}$.

An analysis of these results, when treated by a two-way ANOVA, revealed that there was a significant difference between recovery conditions (Table 2).

A post hoc Tukey test was used to determine which group means differed from each other. There was a significant difference between leg exercise and rest recovery protocols $(P<0.01)$ and a significant

Table 1. Mean(s.d.) lactate values of ten subjects during three modes of recovery, rest, leg exercises and arm exercises, over a 30-min recovery period. Values in $\mathrm{mmoll}^{-1}$, and per cent lactate values shown in bold type $(\mathrm{mean} / \mathrm{max})$

\begin{tabular}{|c|c|c|c|c|c|c|c|c|}
\hline \multirow[t]{2}{*}{ Mode } & \multicolumn{8}{|c|}{ Recovery period (min) } \\
\hline & 0.5 & 2 & 5 & 10 & 15 & 20 & 25 & 30 \\
\hline $\begin{array}{l}\text { Rest } \\
\text { mean } \\
\text { s.d. } \\
\text { Per cent }\end{array}$ & $\begin{array}{r}10.3 \\
2.7 \\
86.6\end{array}$ & $\begin{array}{r}10.9 \\
2.7 \\
91.6\end{array}$ & $\begin{array}{r}11.9 \\
2.0 \\
100\end{array}$ & $\begin{array}{r}11.1 \\
2.3 \\
92.3\end{array}$ & $\begin{array}{r}9.5 \\
2.5 \\
79.8\end{array}$ & $\begin{array}{r}8.0 \\
2.5 \\
67.2\end{array}$ & $\begin{array}{r}6.7 \\
2.3 \\
56.3\end{array}$ & $\begin{array}{r}5.6 \\
1.6 \\
47.0\end{array}$ \\
\hline $\begin{array}{l}\text { Leg exerc } \\
\text { mean } \\
\text { s.d. } \\
\text { Per cent }\end{array}$ & \begin{tabular}{r|}
\multicolumn{1}{c}{ ses } \\
8.7 \\
1.7 \\
87.8
\end{tabular} & $\begin{array}{r}9.9 \\
1.7 \\
100\end{array}$ & $\begin{array}{r}9.7 \\
1.6 \\
98.0\end{array}$ & $\begin{array}{r}8.2 \\
1.3 \\
82.8\end{array}$ & $\begin{array}{r}6.5 \\
1.2 \\
65.7\end{array}$ & $\begin{array}{r}4.6 \\
1.1 \\
46.5\end{array}$ & $\begin{array}{r}3.4 \\
1.0 \\
34.3\end{array}$ & $\begin{array}{r}2.5 \\
0.7 \\
25.3\end{array}$ \\
\hline $\begin{array}{l}\text { Arm exer } \\
\text { mean } \\
\text { s.d. } \\
\text { Per cent }\end{array}$ & \begin{tabular}{r|}
\multicolumn{1}{c}{ ises } \\
10.0 \\
1.8 \\
86.2
\end{tabular} & $\begin{array}{r}10.6 \\
1.9 \\
91.4\end{array}$ & $\begin{array}{r}11.6 \\
1.6 \\
100\end{array}$ & $\begin{array}{r}10.7 \\
1.9 \\
92.2\end{array}$ & $\begin{array}{r}8.2 \\
2.1 \\
70.7\end{array}$ & $\begin{array}{r}6.4 \\
1.4 \\
55.2\end{array}$ & $\begin{array}{r}5.4 \\
1.0 \\
46.6\end{array}$ & $\begin{array}{r}4.3 \\
0.8 \\
37.1\end{array}$ \\
\hline
\end{tabular}


Table 2. Analysis of variance results for lactate values from ten subjects sampled at eight intervals over 30 min under three independent recovery conditions

\begin{tabular}{lcrrr}
\hline & $S S$ & d.f. & MS & $F$ \\
\hline Between interval & \multicolumn{1}{c}{144} & 7 & & \\
Within intervals & 30.5 & 16 & & \\
Between conditions & 27.3 & 2 & 13.63 & $61.9^{*}$ \\
Residual & 3.1 & 14 & 0.22 & \\
\hline
\end{tabular}

$F_{99}(2,14)=6.51$

d.f., degrees of freedom

difference between leg and arm exercises $(P<0.05)$ but there was no significant difference between rest and the arm recovery protocols.

\section{Discussion}

The accumulation of lactic acid in muscle during strenuous exercise may have a significant effect on the development of fatigue ${ }^{9}$ and also on the quality of resumed exercise especially during athletic competition $^{3}$. It is well documented that the removal of lactate following exercise is enhanced if, during recovery, the subject exercises at a reduced intensity $6,7,10$. Although lactate can serve as a substrate for the kidney and heart muscle and is resynthesized back to glycogen in the liver, it can also be utilized by resting and exercising skeletal muscle, 11.

Several studies have investigated the effectiveness of various work intensities during the recovery period on lactate clearance and it was shown that an optimum level of exercise intensity was approximately $40 \% \dot{V}_{\mathrm{O}_{2, \max }}{ }^{4,6}$. Other workers have demonstrated that recovery exercise above the onset of blood lactate accumulation (OBLA), which, when expressed as a heart rate relative to maximum heart rate, is less effective in clearing lactate ${ }^{7}$. In the majority of these studies the mode of exercise incorporated during the recovery phase was similar to that used to produce lactate in the first instance. In our study we were interested in the effectiveness of contrasting modes of exercise on removal rates of lactate. Our results are in broad agreement with others which have shown that active exercise is more effective than passive exercise in removing lactate. However, it was also demonstrated that when lactate was produced through exhaustive arm work, reduced intensity arm work during the recovery phase was less effective in removing lactate than leg exercise. Indeed, in our data there was seen to be no significant difference between light arm recovery exercise and passive rest. This finding, which is in contrast to what was expected, might be explained by the fact that the nature of the arm exercise was discontinuous and it has been demonstrated elsewhere $^{3}$ that lactate removal is more effective during continuous rather than intermittent activity.

These findings are particularly interesting to slalom canoeists and are perhaps more relevant to them during training sessions than during competition for the following reasons. During an event a slalom competitor, who is allowed two competition runs, competes for a maximum of $2 \mathrm{~min}$ during each run. The second run occurs after a minimum time lapse of $2 \mathrm{~h}$ and therefore it would seem reasonable to suppose that lactate clearance has been completed within this time span almost regardless of which recovery protocol is adopted. However, during training bouts slalom canoeists practise on agitated water, often at competition speed, incorporating numerous repetitions and sets of runs. It is suggested that if recovery periods, particularly between paddling sets, incorporate activity off the water involving leg work for a period of approximately $30 \mathrm{~min}$, then the subject would be in better physiological shape for performing more effective subsequent repetition training work.

\section{References}

1 Baker SJ. Post competition lactate levels in canoe slalomists. Br J Sports Med 1982; 16: 112.

2 Karlsson J, Nordesjo LO, Jorfeldt L, Saltin B. Muscle lactate, ATP, and CP levels during exercise after physical training in man. J Appl Physiol 1972; 33: 199-203.

3 Bowen A, Belcastro AN. Comparison of self selected recovery methods on lactic acid removal rates. Med Sci Sports Exerc 1976; 8(3): 176-8.

4 Belcastro AN, Bonen A. Lactic acid removal rates during controlled and uncontrolled recovery exercise. I Appl Physiol 1975; 39: 932-7.

5 Davies CTM, Knibbs AV, Musgrove J. Rate of lactic acid removal in relation to different baselines of recovery. Int $Z$ Angew Physiol 1970; 28: 155-61.

6 Hermansen L, Stensvold I. Production and removal of lactate during exercise in man. Acta Physiol Scand 1972; 86: 191-201.

7 Koutedakis Y, Sharp NC. Lactic acid removal and heart rate frequencies during recovery after strenuous rowing exercise. Br J Sports Med 1985; 19: 199-202.

8 Baker SJ. A study of the behavioural profiles of elite slalom canoeists. PhD thesis, University College of North Wales, 1984.

9 Sahlin K, Harris RC, Nylind B, Hultman E. Lactate content and $\mathrm{pH}$ in muscle samples obtained after dynamic exercise. Am J Physiol 1976; 118: 697-702.

10 Karlsson J. Localised muscle fatigue: role of muscular metabolism and substrate depletion. Exerc Sport Sci Rev 1980; 7: 1-42.

11 Brooks GA, Fahey TD. Exercise Physiology: Human Bioenergetics and its Applications. New York: John Wiley, 1984. 